\title{
Spatial and Temporal Effects of Interleaved Masking in Cochlear Implants
}

\author{
Bom Jun KwOn ${ }^{1}$ And Chris van den HonerT ${ }^{2}$ \\ ${ }^{1}$ Department of Communication Sciences and Disorders, University of Utah, 390 S 1530 E., Salt Lake City, UT 84112, USA \\ ${ }^{2}$ Cochlear Americas, 13059 E. Peakview Avenue, Centennial, CO 80111, USA
}

Received: 25 June 2008; Accepted: 13 March 2009; Online publication: 3 June 2009

\begin{abstract}
Modern cochlear implants utilize interleaved presentation of pulses on different electrodes to avoid physical interference among multiple current fields, yet neural interaction still exists. In the present study, masking was examined with four Nucleus24 users with the banded electrode array in an interleaved masking paradigm, where a probe stimulus was interleaved with a masker stimulus. Spatial and temporal aspects of masking were addressed by fixing the masker at the middle of the electrode array and changing the location of the probe and by testing various stimulation rates: $125,500,2,000$, and $6,410 \mathrm{~Hz}$. In addition, growth of masking (GOM) was assessed by changing the masker level in six steps. Results indicated that masking patterns were generally much wider, regardless of stimulation rate, than those in acoustic hearing. The amount of masking decreased from the peak at the rate of approximately $0.5 \mathrm{~dB} / \mathrm{mm}$ even at the highest masker level. The pattern of GOM with the rates higher than $500 \mathrm{~Hz}$ was different from that observed in previous masking studies, characterized by markedly shallow growth at low masker levels or overall shallow growth. A facilitating effect of the masker (lowering the threshold) was suspected, except for the $125-\mathrm{Hz}$ condition, due to the fibers that were subliminally excited, but not discharged, by the masker with local perturbations of membrane potentials, and were subsequently discharged easily by a lower level probe when the temporal gap between
\end{abstract}

Correspondence to: Bom Jun Kwon · Department of Communication Sciences and Disorders · University of Utah · 390 S 1530 E., Salt Lake City, UT 84112, USA. Telephone: +1-801-5816177; fax: +1-8015817955; email: bjkwon@gmail.com masker and probe was sufficiently short. These results suggest that both refractory characteristics of neurons and neural summation be considered in interleaved stimulation of pulses at high, but clinically relevant, stimulation rates. Overall, the present masking study might provide a basis for models in psychophysics and speech understanding in current cochlear implant systems utilizing high-rate interleaved stimulation.

Keywords: psychophysics, interleaved masking, neural summation, growth of masking

\section{INTRODUCTION}

Masking has been extensively studied in auditory science to show interaction between masker and probe and the frequency selectivity of hearing based on the characteristics of the basilar membrane and beyond. The studies on auditory masking have not only enriched our understanding of the auditory system, but also provided quantitative models that are used for perceptual coding of digital audio signals (such as MPEG Layer-3, or MP3; e.g., Painter and Spanias 1997). In electric hearing through a cochlear implant (CI), the acoustic pathway from the external ear through the cochlea is bypassed, and an electrode array inserted in the cochlear canal delivers electrical stimulation directly to auditory nerve fibers. While masking also occurs in electric hearing, there are a number of differences in the mechanisms of masking between acoustic and electric hearing. In acoustic hearing, the basilar membrane exhibits a mechanical tuning characteristic which is the basis of frequency selectivity of hearing (Moore 1986). As such, frequency- 
resolving mechanisms of the auditory system are studied with acoustic masking, and the results are often associated with the auditory filter, where processing of information within each filter is presumed to be independent (Moore and Glasberg 1986a). Such mechanical tuning does not exist in electric hearing, however, without the contribution of the basilar membrane, and the "selectivity" of auditory channels, if any, is influenced by the degree of overlap in neural populations excited by different electrodes. (Eddington et al. 1978; Shannon 1983; Tong and Clark 1986).

In masking experiments that address frequency selectivity of the auditory system, the masker is presented either simultaneously with the probe or nonsimultaneously (usually prior to probe stimulation; forward masking). Masking patterns in acoustic hearing are similar in both paradigms, except that those of forward masking exhibit a sharper tuning characteristic due to the effect of lateral suppression (Shannon 1976). In contrast, forward masking has primarily been studied to address the selectivity of hearing in electric hearing (Boex et al. 2003; Chatterjee and Shannon 1998; Kwon and van den Honert 2006; Nelson et al. 2008; Shannon 1983; Tong and Clark 1986). Simultaneous masking has mostly been tested to examine the spatial spread of excitation with specific electrode configurations through physiological measurements (White et al. 1984; Bierer and Middlebrooks 2004; Stickney et al. 2006). Behavioral simultaneous masking data have not been reported in the literature partly because perceptual consequences of simultaneous stimulation on multiple channels vary drastically depending on the phase relations among the stimuli. It has even been suggested that loudness summation rather than simultaneous masking might be a better indicator for channel spread (i.e., functional selectivity of hearing in the context of the present paper) when measured behaviorally (Shannon 1983). It is difficult to consider simultaneous masking in electric hearing as a counterpart of simultaneous masking in acoustic hearing because superposition of two current fields often results in an excitation pattern that is widely different from that of each current field presented alone. Consequently, this new excitation pattern might be perceived as an unintended distortion, whereas the interaction between two acoustic tones falling into one auditory filter ("unresolved" components) still contributes to the overall perceptual consequences in an orderly manner.

Virtually all CI systems presently used adopt pulsatile interleaved stimulation, in which pulses on multiple channels are stimulated with a small temporal gap (from a few tens to hundreds of microseconds), so that stimulation on multiple channels can be delivered in the same time window while avoiding the interactions of current fields. Therefore, simultaneous masking in electric hearing can be studied in the context of interleaved stimulation rather than in the strict sense of simultaneous stimulation. Despite the prevalent use of interleaved stimulation in clinical applications, masking in interleaved stimulation (throughout this paper it will be referred to as "interleaved masking") has not been studied as widely as it deserves, as only limited data are available in the literature on this type of masking (Tong and Clark 1986; Zeng et al. 2005). Interleaved masking is different from forward masking in that there is a temporal overlap of the masker and probe presentation. It is also different from simultaneous masking in that the pulses of masker and probe are temporally interleaved with each other. Therefore, the present study aimed to investigate interleaved masking through a systematic exploration of the following stimulation parameters: (1) masker-probe separation on the electrode array (to obtain the masking pattern), (2) the level of masker (to measure the growth of masking), and (3) the masker-probe gap (to investigate the effect of stimulation rate). While the first two parameters have frequently been examined in previous forward masking studies, it is still worthwhile to measure them in the context of interleaved stimulation. The examination of the third parameter, which has not been studied in a psychophysical experiment, was motivated to make the study findings more relevant to clinical applications. As CI systems offer a wide range of stimulation rates in clinical fitting, it is critical for us to understand the nature of masking among channels that occur when a multi-channel stimulus is delivered through interleaved stimulation at various rates.

\section{METHODS}

\section{Subjects and equipment}

Four postlingually deafened adult CI users participated in the present study. All subjects were implanted with the Nucleus ${ }^{\circledR}$ CI24M device (straight, banded electrode array), and each had at least 2 years experience with their implant prior to testing. They were the same subjects as in our forward masking study (Kwon and van den Honert 2006), and the data were collected within 6 months after the completion of that study. Detailed subject information was included in Table 1 in our previous report (Kwon and van den Honert 2006). The use of human subjects in this study was reviewed and approved by the Western Institutional Review Board. Custom software controlled the stimuli and administered testing sessions. The apparatus was described in detail in the previous paper. 


\section{Stimulation parameters}

In the present study, bi-phasic, charge-balanced pulse trains were used in a monopolar electrode configuration. The phase duration of each pulse was $25 \mu$ s and the interphase gap was $8 \mu \mathrm{s}$. The masker was always presented on electrode 10, and the probe was presented on one of the following electrodes: 2, 8, 10, 12, and 18. As shown in Figure 1, the durations of masker and probe were 500 and $50 \mathrm{~ms}$, respectively. The probe was placed in the center of the masker duration (225 ms after the onset of the masker), where the neural discharge from the masker was considered to be in a steady state. While it has been observed that a peripheral "over-shoot" or "undershoot" effect in the discharge rate at the vicinity of temporal edges of onset or offset of stimuli may affect the measurement of masking in electric hearing (Smith et al. 2003; Zeng et al. 2005), the present study primarily sought to examine masking without such temporal edge effect. The stimulation rate of both masker and probe was one of the following: 125,

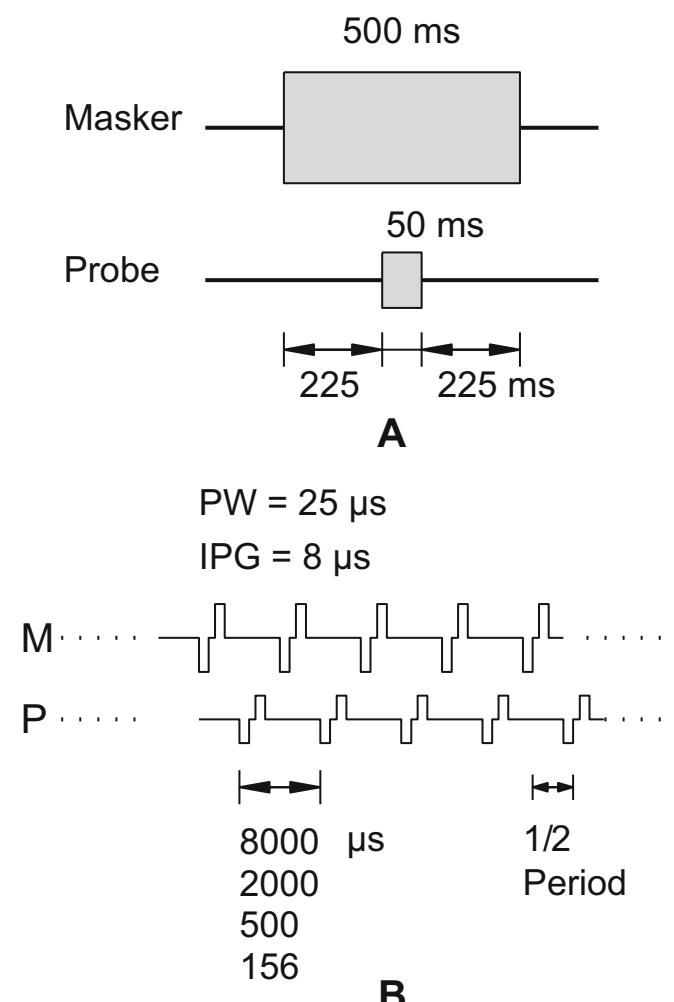

FIG. 1. Schematics depicting the timing of the pulse trains. (A) overall view: The probe (duration, $50 \mathrm{~ms}$ ) is presented $225 \mathrm{~ms}$ after the onset of the masker (duration, $500 \mathrm{~ms}$ ) (B) pulse layout: The period of masker pulse train was 8,000, 2,000, 500, and $156 \mathrm{~ms}$, for the $125,5,000,2,000$, and $6,410 \mathrm{~Hz}$ condition, respectively. Individual pulses always had a phase duration (PW) of $25 \mu$ s and an interphase gap (IPG) of $8 \mu \mathrm{s}$. The probe and masker pulses were interleaved at symmetrical timing; therefore, the interpulse period was a half during the probe presentation.
500, 2,000, 6,410 Hz. The probe was interleaved with the masker with symmetrical timing (cf. Fig. 1B). For example, for the $2,000-\mathrm{Hz}$ condition, the interpulse period of the masker alone was $500 \mu \mathrm{s}$, but it was $250 \mu$ s during the interleaved presentation. Several current levels of the masker were chosen based on each subject's loudness ratings: L1, L2,... L6, where L1 corresponded to the threshold level of the masker (barely audible; see below), L4 was the "comfortable" level (medium level), and L6 was the "very loud" level (which might be slightly higher loudness than that of the clinical C-level, which tends to be set conservatively). The other levels (L2, L3, and L5) were determined so that those six masker levels could evenly span the entire range of perceptual loudness. The current level of L1 was established by measuring the threshold of a 500-ms pulse train for each of the four stimulation rates with an adaptive procedure (Levitt 1971) in a three-alternative-forced-choice paradigm with a two-down-one-up criterion, corresponding to the level with $70.7 \%$ correct response. Then the levels L2 through L6 for the $125-\mathrm{Hz}$ condition were established as described above, and these levels were used as references for loudness rating of other stimulation rates. Since the loudness rating was arbitrary, there was no expectation that L3 of one subject would be the same as L3 of other subjects, but the ratings were consistent within each subject across different stimulation rates. While the currents did not increase evenly from L1 to L6, as seen on the abscissa of the graphs in Figure 5, subjective loudness and the amount of masking were roughly evenly distributed (as seen in Fig. 4).

\section{Procedure and notation of results}

Stimulation current in this experiment was controlled in terms of "current level (CL)," which is an integer ranging from 0 to 255 in a logarithmic scale. In the Nucleus-24 system, zero CL is equal to $10 \mu \mathrm{A}$ and an increase of $1 \mathrm{CL}$ corresponds to approximately a $0.175-\mathrm{dB}$ increase of the current. The $\mathrm{CL}$ unit is widely used in clinical fitting software, and the relation between CL and actual current in $\mu \mathrm{A}$ is described in our previous paper (Kwon and van den Honert 2006). The masked thresholds (and the quiet thresholds as described above) were measured using an adaptive, three-alternative forced-choice procedure (two-down/one-up). The masker was present in all three intervals, whereas the probe existed in only one interval. Each interval was separated by a $400-\mathrm{ms}$ silence gap. The subject's task was to identify the "different" interval. The level of the probe was adjusted according to subject response, ultimately converging on $70.7 \%$ correct (Levitt 1971). The adjustment step size was initially 5 CLs (corresponding to $0.88 \mathrm{~dB}$ ) and 
was reduced to $2 \mathrm{CLs}(0.35 \mathrm{~dB})$ after six reversals. Each run contained 14 reversals, and the threshold was calculated as the mean of the probe level in the last eight reversals. Each condition was repeated three times for further statistical analyses. In addition, absolute detection thresholds without the masker were measured for all probe stimuli through the same procedure.

There has been a debate as to which scalelogarithmic or linear-should be used when specifying stimulation currents in electric hearing and the plotting of results. The rationale for the linear scale is based on the physiological observation that the linear current is directly related to the response of the nerve population, which is attributed to the lack of peripheral compression in electric hearing (Zeng 2004). For this reason, the linear current scale might be preferable when comparing two currents within the same stimulation setting (i.e., in one subject or in the same electrode configuration). For example, growth of masking (the increase of masking as a function of masker level) might be properly represented in a plot of linear $\mu \mathrm{A}-\mu \mathrm{A}$ axes, as is typically done in the literature (e.g., Chatterjee and Shannon 1998; Nelson and Donaldson 2001). On the other hand, a logarithmic scale might be a sensible choice if normalization of values is necessary when comparing currents over a wide range (different subjects or electrode configurations). In addition, it might be reasonable to use a logarithmic $(\mathrm{dB})$ scale when describing masking patterns to facilitate the comparison with acoustic masking data. Therefore, with the rationale and technical characteristics described above, results in this paper are represented in a $\mathrm{dB}$ scale. The exception is the growth of masking functions and related figures plotted in a linear $\mu \mathrm{A}$ scale (Figs. 5, 8, and 9).

\section{RESULTS}

Description of masking data and statistical analyses

Figure 2 displays the thresholds of the probe across electrode locations with different stimulation rates. Each panel displays the data of one subject. The thresholds, in general, decreased as stimulation rate increased, indicating that temporal integration takes place, i.e., the more pulses delivered to the auditory nerve, the lower current required for detection of the probe. A repeated measures of analysis of variance (ANOVA) indicates that the effect of stimulation rate was significant $(F(3,9)=43.6, p<0.001)$, and the effect of probe electrode was not $(F(4,12)=1.26, p=0.34)$. In order to assess the effect of stimulation rate on the threshold, the data from all subjects were restructured

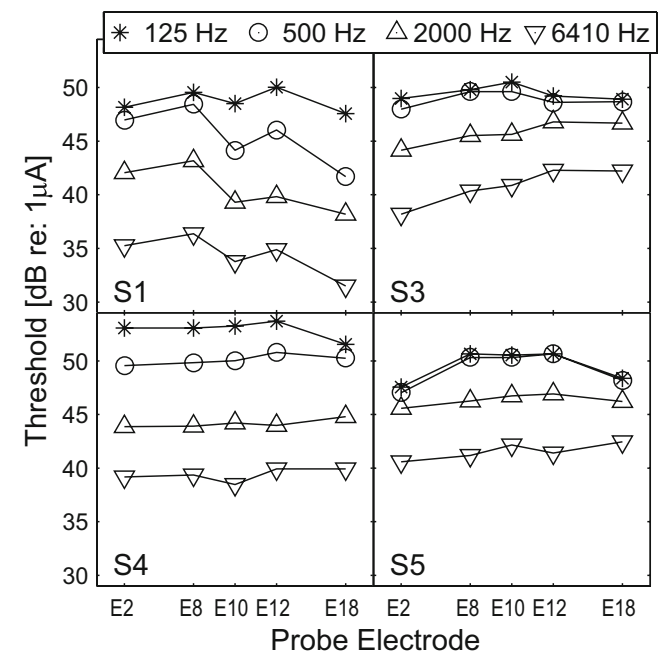

FIG. 2. Probe thresholds across electrode locations with different stimulation rates as a parameter.

and plotted in one panel as a function of stimulation rate as displayed in Figure 3. A linear regression analysis, done over the range of the stimulation rates tested, indicated a decrease of $2.0 \mathrm{~dB}$ in threshold per octave with the stimulation rate $\left(R^{2}=0.74, p<0.0001\right)$. In a study by Middlebrooks (2004), the detection thresholds decreased with a rate of $2 \mathrm{~dB}$ per octave for the rates above $1,000 \mathrm{~Hz}$ (from a visual inspection of Figure 10 in that paper) but were almost unaffected by the stimulation rate at lower rates. In fact, the same trend is found in two subjects (S3 and S5) of the present study, where the thresholds are similar for the 125- and $500-\mathrm{Hz}$ conditions (cf. Fig. 2). While it is unclear why this trend appears in only two subjects, the data of the other subjects (S1 and S4) suggest that the temporal window during which the peripheral information is integrated at the auditory cortex might be longer in some human subjects. In other words, while the temporal window of $1 \mathrm{~ms}$ was suggested by Middlebrooks (2004; p. 461) from the data obtained physiologically in a guinea pig model, it might be different or could show a variability in humans.

The full data set shown in Figures 4 and 5 represents masking as a function of spatial and temporal relations between masker and probe. The panels are arranged for each subject (row) and each stimulation rate (column). Figure 4 displays masking patterns (i.e., masking as a function of probe location with a fixed masker location), and Figure 5 displays the growth of masking (GOM) functions (i.e., masking as a function of the masker level). The amount of masking in Figure 4 is the difference in threshold in dB. Threshold shifts in Figure 5 indicate the difference in $\mu \mathrm{A}$. As mentioned in the "Methods" section, the choice of scale-masking patterns in a log scale 


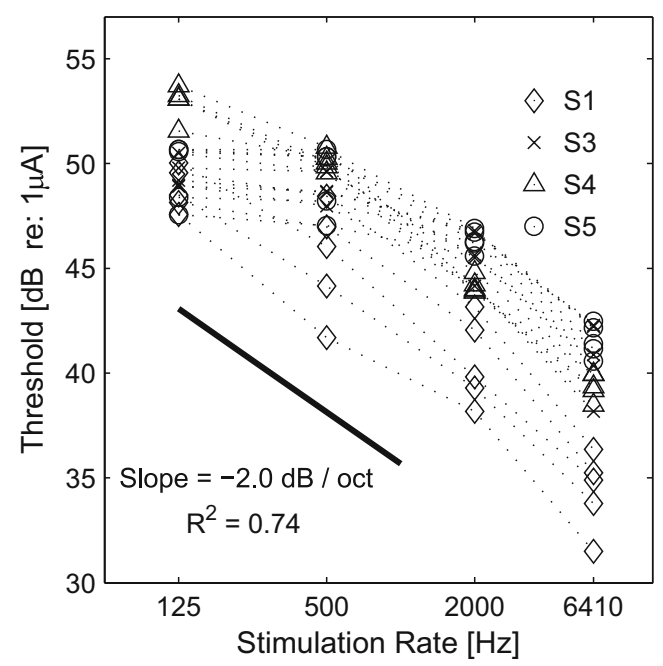

FIG. 3. Probe thresholds in Figure 2 re-plotted as a function of stimulation rate.

and GOM functions in a linear scale-was made mainly to facilitate discussion: masking patterns in Figure 4 were intended to compare with those in acoustic masking (always in a $\mathrm{dB}$ scale), whereas the $\mu \mathrm{A}-\mu \mathrm{A}$ axes in GOM functions in Figure 5 were from the convention in the literature (e.g., Chatterjee and Shannon 1998; Nelson and Donaldson 2001).

A large individual variability was observed with regard to the effects of stimulation rate, spatial selectivity, and GOM. Greater masking was observed in S1 (with the peak of 10-15 dB with $500 \mathrm{~Hz}$ and faster stimulation rate conditions) than other subjects, where only modest amounts, not greater than $8 \mathrm{~dB}$, were observed. Small amounts of masking (about $2 \mathrm{~dB}$ or less) in S3 in fast stimulation rate conditions $(2,000$ and $6,410 \mathrm{~Hz}$ ) were surprising, along with the absence of the peak in masking patterns. Regarding the question whether (or how) the increase of masker level influences the width of masking patterns, a visual inspection only led to mixed observations: sometimes the peak was broader (e.g., S4/125 Hz, S5/125 Hz, $\mathrm{S} 5 / 6410 \mathrm{~Hz})$ or about the same $(\mathrm{S} 1 / 500 \mathrm{~Hz}, \mathrm{~S} 1 /$ $6410 \mathrm{~Hz}, \mathrm{~S} 3 / 125 \mathrm{~Hz}, \mathrm{~S} 5 / 500 \mathrm{~Hz}$ ) as the masker level increased. With regard to GOM functions (Fig. 5), a greater slope was expected on the E10 condition (the "on-channel" condition where the sites of masker and probe stimulation were the same). Yet, only a few conditions followed such expectation (e.g., S1/ $6410 \mathrm{~Hz}, \mathrm{~S} 5 / 500 \mathrm{~Hz}, \mathrm{~S} 5 / 2000 \mathrm{~Hz}$ ), and more often, the E8 and E12 conditions clustered together with the

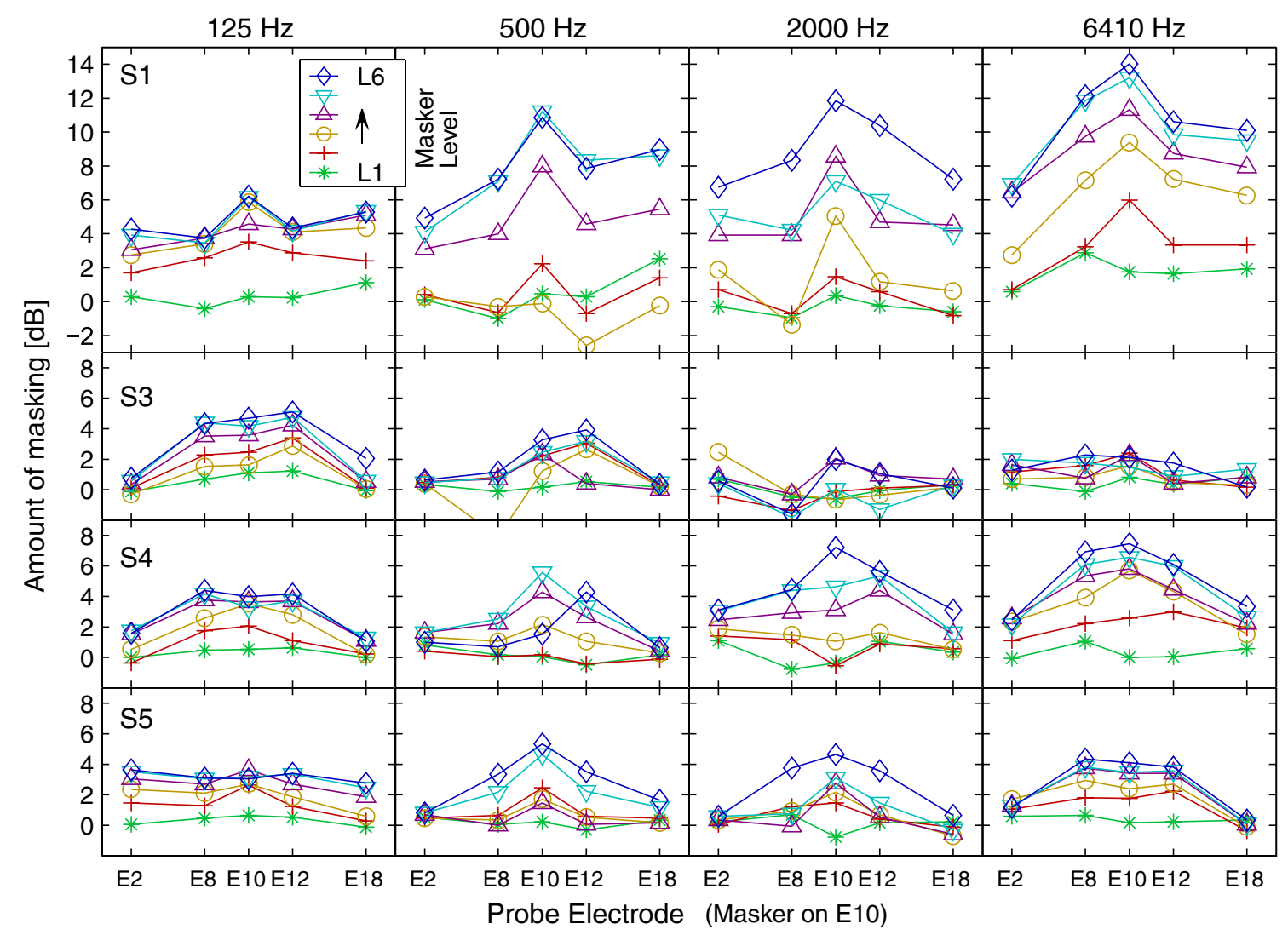

FIG. 4. Masking patterns (amount of masking as a function of probe location with a fixed masker location at E10). Amount of masking was calculated as the difference in thresholds with and without the masker in CL then converted to dB. Each row corresponds to each subject and each column corresponds to each stimulation rate used. 

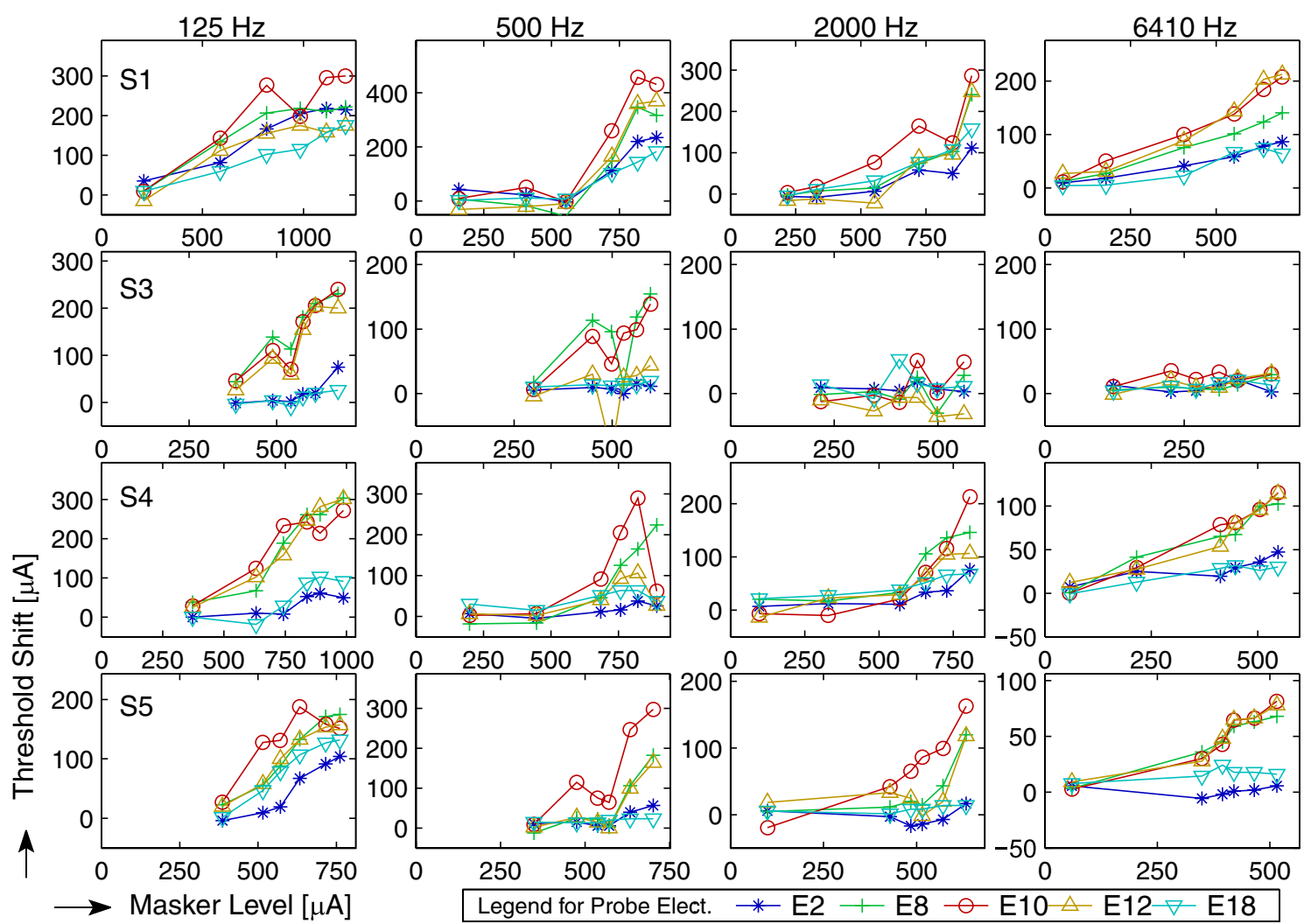

FIG. 5. Growth of masking (GOM) functions for each subject and stimulation rate. Amount of masking is represented here by threshold shift (the difference in threshold in $\mu \mathrm{A}$ ).

E10 condition (e.g., S3/125 Hz, S4/125 Hz, S4/ $6410 \mathrm{~Hz}$, and $\mathrm{S} 5 / 6410 \mathrm{~Hz}$ ), suggesting a broad spatial pattern of masking. In the following two sections, quantitative analyses are provided in detail regarding the two facets of masking-spatial selectivity and GOM.

\section{Spatial selectivity}

To analyze the spatial selectivity, a metric, "spatial attenuation of masking" was defined in the present study. This represents the decrease of masking in $\mathrm{dB}$ per millimeter from the peak under the presumption that the decrease is linearly related to the distance from the peak. A higher value of spatial attenuation indicates the amount of masking decreases rapidly from its peak, and vice versa. Zero spatial attenuation indicates a flat masking pattern. The purpose of this modeling was to quantify the sharpness of masking patterns and to compare them across conditions. While an alternative modeling based on the width of a curve fit to the data could also be possible, it was not used because the sum of squared errors of the curve fitting was generally higher. From each masking pattern in Figure 4 (a total of 96-four rates by six masker levels for four subjects), the attenuation value $(\mathrm{dB} / \mathrm{mm})$ was obtained in the following way. First, 15 data points in each pattern (five probe locations by three repeats) were analyzed by one-way ANOVA, with probe location as the factor, to check whether there was a significant effect of probe location. If the $p$ value was greater than 0.02 , the masking pattern was considered "flat," and a zero attenuation value was assigned (for example, most L1 conditions). If not, an attenuation value was obtained by a linear regression of those 15 data points consisting of three points at zero distance (E10), six at $1.5 \mathrm{~mm}$ (E8 and E12) and another six at $6 \mathrm{~mm}$ (E2 and E18; note: electrode spacing in the Nucleus-banded electrode array is $0.75 \mathrm{~mm}$ between the centers of electrode bands). Figure 6 displays the mean of spatial attenuation for each subject and the across-subject mean averaged across probe conditions as a function of masker level. The higher the level of the masker, the steeper the masking pattern. At the highest masker level (L6), the attenuation was on average almost $-0.5 \mathrm{~dB} / \mathrm{mm}$. Caution should be exercised when interpreting this curve, as the average on each ordinal scaling was made across subjects (as mentioned earlier, one subject's loudness scale cannot be objectively related to other subject's scale). The dependency of the sharpness on the masker level was further analyzed in the abscissa that allowed an objective comparison across subjects. Figure 7 shows scatter plots of spatial 


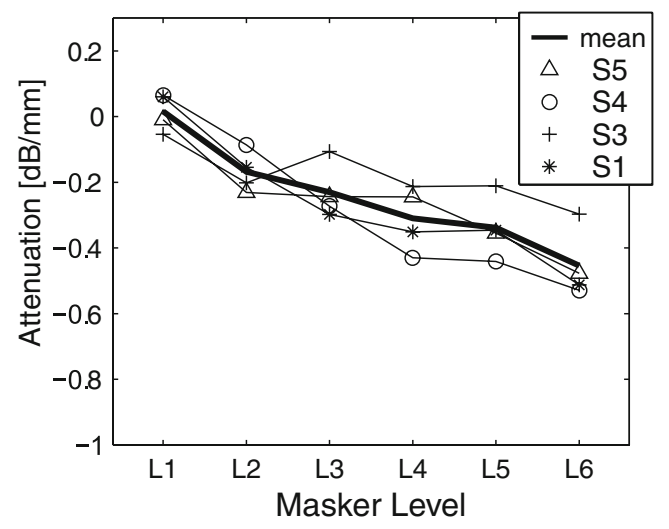

FIG. 6. Spatial attenuation $(\mathrm{dB} / \mathrm{mm})$ for each subject and the across-subject mean as a function of masker level. The higher the level of the masker, the steeper the masking pattern.

attenuations for each subject as a function of masker level normalized by $\mathrm{dB}$ percent dynamic range $\left(\% \mathrm{DR}_{\mathrm{dB}} ; 100 \frac{d B_{M}-d B_{T H}}{d B_{M A X}-d B_{T H}}\right.$, where $d B$ indicates the current specified in $\mathrm{dB}$, the subscripts MAX, TH, and $\mathrm{M}$ indicate maximally tolerable level, threshold, and the masker level, respectively), along with the results of linear regression to assess the dependency of the increased steepness with a masker level. All subjects but S3 showed a clear pattern of increased steepness with an increase of the masker level, as the regression analyses yielded significant $p$ values, as indicated in Figure 7. However, despite the statistical analysis, an application of this trend should still be guarded because, in a number of instances, the attenuation was near zero (large spatial spread of masking) at high masker levels in all subjects.

\section{Growth of masking}

Growth of masking (GOM) functions are typically fit by a linear function, and the slope $(\mathrm{dB} / \mathrm{dB}$ in acoustic hearing and $\mu \mathrm{A} / \mu \mathrm{A}$ in electric hearing) is interpreted as the masker's effectiveness across different levels. Although a visual inspection of the GOM functions in Figure 5 suggests that a curve fitting by a nonlinear function might be suitable, a linear fitting was initially made for the comparison with previous data. That is, a total of 80 slopes in $\mu \mathrm{A} / \mu \mathrm{A}$ (from Figs. 5; four rates by five probe locations for four subjects) were obtained from linear regression and were analyzed by a repeated measures of ANOVA to determine statistical significance. There was a significant effect of probe location $(F(4,12)=62.2, p<0.001)$, but the effect of stimulation rate was not significant $(F(3,9)=2.619, p=$ $0.115)$. The interaction between probe location and stimulation rate was also not significant $(F(12,36)=$ $1.62, p=0.13)$. Figure 8 shows the mean of GOM slopes for each subject and the across-subject mean as a function of probe electrode. The slopes of S3 were the smallest, due to the small effect of masker level seen in high stimulation rates $(2,000$ and $6,410 \mathrm{~Hz})$. The E10 condition ("on-channel" masking) produced the steepest mean slope $(0.3359)$. The slopes at E8 and E12 were still comparable to the slopes at E10 (0.2521 and 2823, respectively); but those at E2 and E18 were markedly shallower (0.1009 and 0.0972, respectively), which might be considered as "offchannel" masking.

In addition, GOM was examined in detail for the on-channel masking condition (E10 condition). In this analysis, a nonlinear function was used to model the GOM functions. The effect of stimulation rate was of particular concern, as the temporal separation

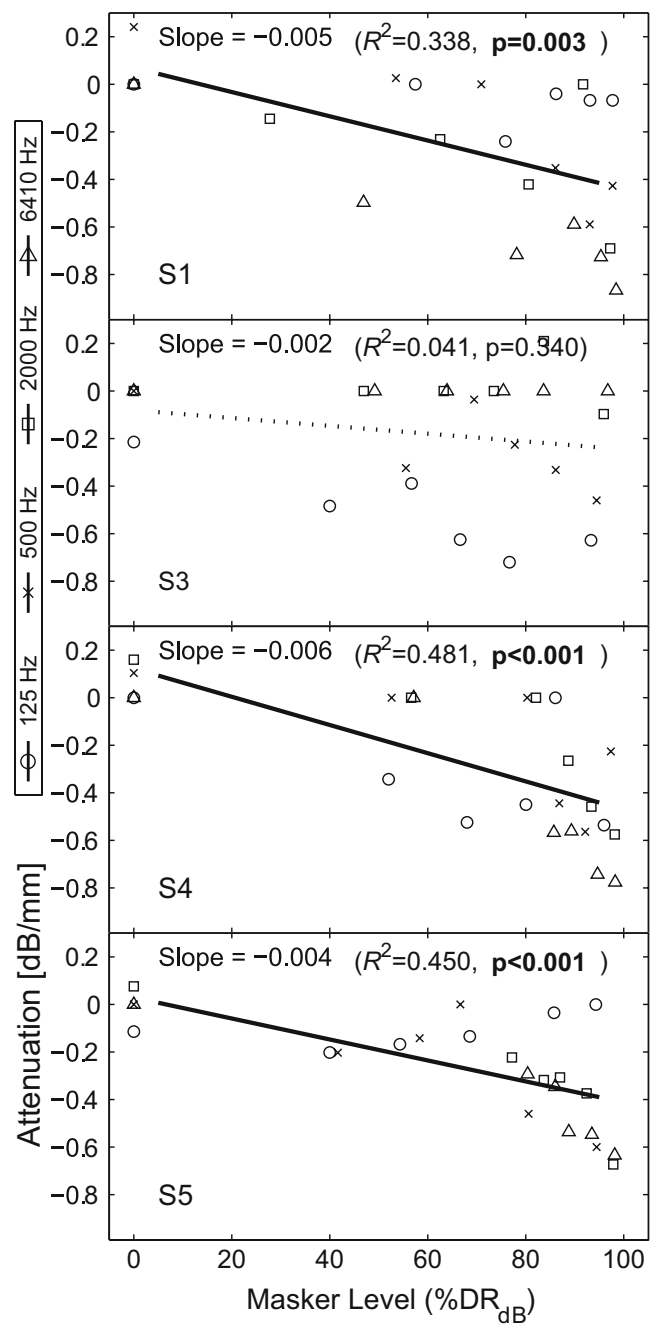

FIG. 7. Scatter plots of spatial attenuations for each subject as a function of masker level in $\mathrm{dB}$ percent dynamic range $\left(\% D R_{\mathrm{dB}}\right)$. Results of linear regression are also shown to display the trend between spatial attenuation and masker level. All subjects but S3 showed a clear pattern of increased steepness with an increase of the masker level. It should still be noted that, despite the statistical trend in a number of instances, the attenuation was near zero (large spatial spread of masking) at high masker levels in all subjects. 


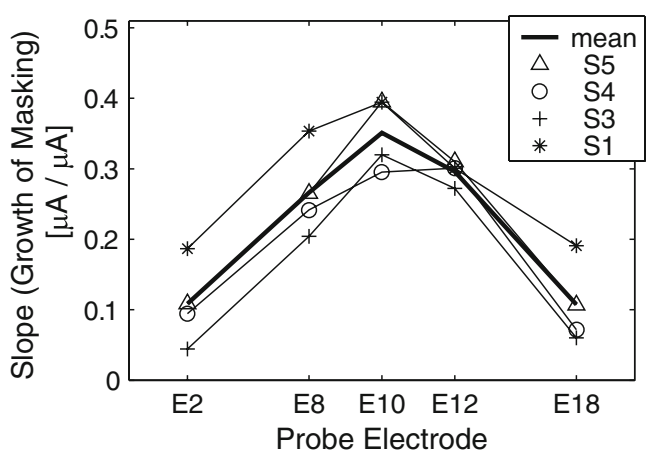

FIG. 8. GOM slopes for each subject and the across-subject mean as a function of probe electrode. The slopes are the greatest with the probe presented on E10 (on-channel masking).

between masker and probe decreased as the stimulation increased, potentially affecting the growth of masking. Furthermore, unlike the spatial selectivity that could vary greatly from one subject to the next due to heterogeneous patterns of nerve survival, a temporal effect might vary to a lesser degree unless degeneration of the auditory nerve or the central nervous system was suspected. Therefore, in order to reveal an effect of stimulation rate, the GOM data of all subjects in the E10 condition were regrouped for each stimulation rate and a scatter plot was drawn, as seen in Figure 9. As seen in Figure 5, some GOM functions show a very shallow growth at lower masker levels, and then grow more steeply as the masker level increases. Therefore, it is not proper to plot the functions across subjects by the absolute current. Instead, normalized currents by the dynamic range are used in Figure 9, as specified by percent dynamic range $\left(\% \mathrm{DR} ; 100 \frac{I_{\mathrm{M}}-I_{\mathrm{TH}}}{I_{\mathrm{MAX}}-I_{\mathrm{TH}}}\right.$, where $I$ is current in the $\mu \mathrm{A}$ unit, the subscripts MAX, TH, and $\mathrm{M}$ indicate maximally tolerable level, threshold, and the masker level, respectively). In the present study, we propose a knee-point model, where masking is presumed to be zero and does not grow until the masker level reaches a certain level (the rationale of the knee-point model is provided in the "Discussion" section). For each panel in Figure 9, given a knee point, a linear regression was done with the points above it, and a flat horizontal line with zero masking was modeled below it. Then the sum of the squared residuals (SSR) for this fitting was calculated for the entire points. The "best" fit was obtained that produced the least SSR after the knee point was swept from $0 \%$ to $80 \%$. The four best results displayed in Figure 9 were fit with good significance $(p<0.001)$. As expected from Figure 5, the 125- and 6,410- $\mathrm{Hz}$ conditions yielded the best fits with the knee point of $0 \%$-i.e., a linear function without a knee point. The other conditions required nonzero knee points for the best fit $-20.5 \%$ and $48.1 \%$, for the $500-$ and $2000-\mathrm{Hz}$ condition, respectively. These analyses indicated the following.

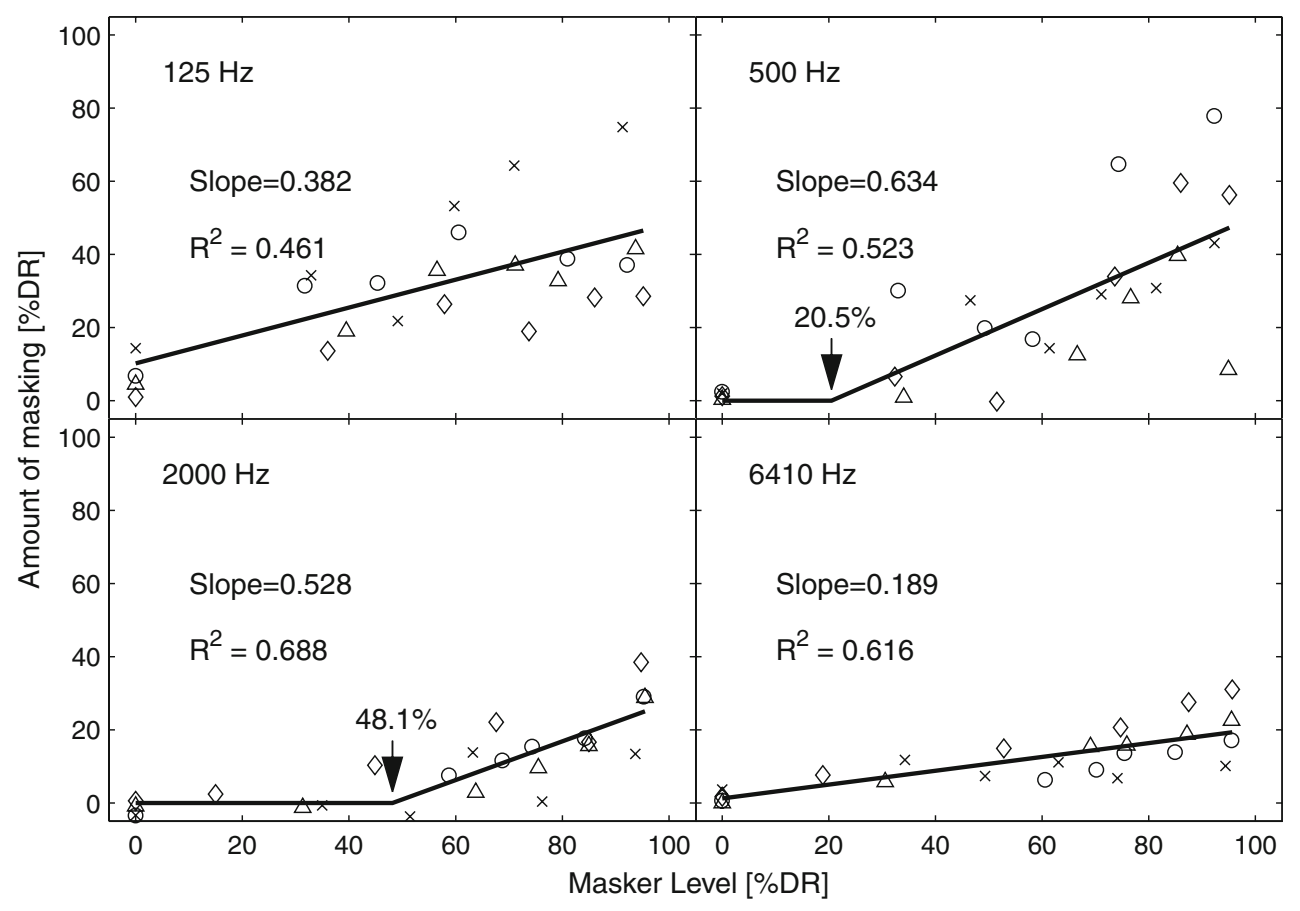

FIG. 9. GOM functions arranged for each stimulation rate as a function of masker level in percent dynamic range (\%DR), collapsing the data across subjects. The function was fit by two lines (a flat line and increasing from a knee point). For each knee point, which was iterated from $0 \%$ to $80 \%$, the data were fitted to the lines. The knee point and corresponding slope that produced the least sum of squared residual (SSR) were taken as the parameters for the "best fit" and indicated here. The knee-point fitting was successful in the two rate conditions (500 and 2,000 Hz). 
For the $125-\mathrm{Hz}$ condition, with a long masker-probe interpulse interval (4 ms), simple linear regression was successful, and the mean slope (0.382) was roughly comparable to the slopes of previous forward masking data (Kwon and van den Honert 2006). However, a complex mechanism might underlie for higher stimulation rates because a knee-point model adequately fit the data in the 500- and $2,000-\mathrm{Hz}$ conditions (i.e., masking does not always grow linearly as the masker level increases). Although a linear model appeared to be successful for the $6,410-\mathrm{Hz}$ condition, the mean slope was markedly small (0.189) for on-channel masking, which required further discussion (see below).

\section{DISCUSSION}

Possible effects of neural summation implied by shallower GOM slopes

The GOM slope of 1 in acoustic hearing (Hawkins and Stevens 1950) indicates that as the masker level increases, masking grows by the same amount. A shallower slope means that a given increase in the masker level requires a smaller increase in the probe level. In other words, as the level increases, the masker provides less effective masking. This could take place either (1) when the masker stimulus does not excite the same neural population affected by the probe (such as off-frequency masking) or (2) when the masker and probe do not simultaneously excite the neural population (such as forward masking). The forward masking slope in acoustic hearing decreases as the masker-probe interstimulus delay becomes larger (Jesteadt et al. 1982). Indeed, similar to acoustic hearing, the slope of GOM in on-channel simultaneous masking in electric hearing has been found to be close to 1 (Zeng et al. 2005) and less than 1 in forward masking (Chatterjee and Shannon 1998; Nelson and Donaldson 2001). Although the presentation of the masker and probe in the present experiment was not truly simultaneous, the effect of the masker was expected to resemble that of simultaneous masking because the interpulse (interleaved) period between masker and probe was fairly small $(\leq 1 \mathrm{~ms})$, as shown in Figure 1, except for the $125-\mathrm{Hz}$ condition (4 ms). Even in forward masking, a maskerprobe delay of $1 \mathrm{~ms}$ is often sufficient to make the slope approach one (Nelson and Donaldson 2001). Nevertheless, the slopes in the present study measured for delays less than $1 \mathrm{~ms}$ were far smaller than 1 . Particularly striking is that the slopes in the present study were even smaller than those obtained in the previous forward masking experiment with a maskerprobe delay of $20 \mathrm{~ms}$ with the same subjects (Kwon and van den Honert 2006) - about 0.4 or greater when the masker and probe electrodes were closely located. This appeared to be attributed to by a shallow growth of masking with maskers at low levels, suggesting a possible effect of neural summation.

A phenomenon of negative masking, or "facilitation" by a masker, can sometimes be observed in electric hearing, i.e., detection threshold is lower when the masker is present than in quiet, as seen in both behavioral (Eddington et al. 1978; Nelson and Donaldson 2001; Tong and Clark 1986; Zeng et al. 2005) and physiological measurements (e.g., Middlebrooks 2004). In those studies, the masker was commonly at a low (almost subthreshold) level. This phenomenon is based on the effect of neural summation. When a masker delivers stimulation to a nerve fiber just below its threshold so that the masker alone does not generate an action potential, subliminal changes (or perturbation) in the cell membrane potential, due to local depolarization, can still take place. If a probe arrives at the neuron before this subliminal response fades away, an action potential can be triggered at a lower level. When the masker is presented at a substantially higher level than the threshold, since action potentials are already generated in a group of nerve fibers which are less responsive to the probe as they are in the refractory stage, this phenomenon is more difficult to occur. Nevertheless, although negative masking is not observed as a separate phenomenon, neural summation might still be in effect with a suprathreshold masker. The knee-point model considered in the "Results" section provides indirect evidence of this, as masking did not tend to grow until the level of the masker was substantially higher than the threshold in the two conditions of stimulation rates $(500$ and $2000 \mathrm{~Hz})$. When the masker was at a low to moderate level, some fibers were not discharged by the masker alone but could be discharged when both masker and probe were present. In other words, although a group of fibers were already discharged by the masker and thus less responsive to the probe, another group of fibers that were in the subliminal state would probably be able to respond to a lower level probe. Therefore, these fibers could provide sufficient information for detection of the probe. This would no longer be the case when the masker exceeded a certain level and a predominant proportion of neural population of interest was activated (thus no fibers are available for probe detection).

As physiological measurements on the temporal course of neural summation in the auditory nerve are rare in the literature (Cartee et al. 2006), it is not clear how long the perturbation of membrane potential persists, although it is unlikely to persist beyond the relative refractory period. Therefore, it would be reasonable to discard the effect of neural summation in the $125-\mathrm{Hz}$ condition and masking could be believed to be governed primarily by refractory nature 
of neurons (and possibly a central-level mechanism similar to that in forward masking). In contrast, it is likely that masking was influenced by neural summation in the higher stimulation rate conditions, as the knee-point model successfully fit the data. Finally, it is surprising that the knee point was not found in the $6,410-\mathrm{Hz}$ condition because it is difficult to reason that neural summation disappeared at this rate if it existed at lower rates. We speculate that neural summation was still in effect and accounted for the considerably shallow slope of GOM at this rate. Overall, we admit that this analysis did not exclusively prove that neural summation was in effect with a suprathreshold masker. Yet, it should be noted that GOM in interleaved masking at a relatively high stimulation rate could not be explained merely with the refractory nature of the masker and the knee-point model considering that the contribution of neural summation adequately accounted for the data. Neural summation is particularly important to consider in interleaved masking because the masker and probe pulses are likely to interact more at the periphery than in forward masking. In summary, the present data suggest that both effects-neural summation and refractorinessshould be considered when examining the interaction between pulse trains in an interleaved presentation with a short interpulse interval.

Implications for speech processing strategy and other models in electric hearing

Spatial attenuation of masking was about $-0.5 \mathrm{~dB} / \mathrm{mm}$ at the highest masker level (cf. Figs. 6 and 7). This corresponds to $-0.38 \mathrm{~dB}$ per electrode spacing (electrode spacing in the Nucleus-banded array is $0.75 \mathrm{~mm}$ ); i.e., the amount of masking decreases only by $3.2 \mathrm{~dB}$ at the sites of nine electrodes apart from E10 (to E1 or E19). In addition, there appears to be a large individual variability due to the uneven neural survival profile in each individual. In a typical frequency allocation for speech processing of the Nucleus system, E10 represents the frequency band of 2,100-2,300 $\mathrm{Hz}$, and E1 and E20 may represent the lowest frequency band (around $200 \mathrm{~Hz}$ ) and the highest band (approximately $7,000-8,000 \mathrm{~Hz}$ ). This shallow spatial attenuation of masking (a decrease by about $3 \mathrm{~dB}$ in this wide frequency span - almost the entire range of speech processing) is substantially broader than that of acoustic hearing, not only in normal hearing (Egan and Hake 1950) but also in individuals with sensorineural hearing loss (Moore and Glasberg 1986a, b). While the present study primarily addresses the detection, not discrimination, of a stimulus in the presence of a masker, the present results reveal the degree of potential channel interactions involved in interleaved stimulation that is widely used in the current CI systems. This might be related with poor frequency resolution in CI systems measured in a variety of methods, such as frequency rippled noise (Henry and Turner 2003) and support the observation that loudness summation in electric hearing is largely independent of the sites of stimulation (McKay et al. 2003). Also, the results of the present study suggest that the summation effect be considered in a loudness model, such as McKay et al.'s (2003).

In clinical applications of the Nucleus CI system, the temporal separation between successive pulses typically falls into the range where neural summation might occur (approximately a few hundred microseconds). Therefore, the effect of neural summation should be carefully considered to assess the impact of masking on the performance of CI users. It should also be noted that the present study measured masking by a steady-state masker; had the probe been placed toward the temporal edge of the masker, which might often be the case in clinical applications, the overshoot or undershoot phenomenon might have further altered the result patterns (particularly the GOM functions even more so than expected in acoustic hearing), as mentioned in the "Methods" section. A further study might properly address this aspect. Finally, the present study (or an advanced study) could serve as a psychophysical model for a speech processing strategy based on perceptual masking that was recently proposed and tested [Psychoacoustic ACE (PACE); Buchner et al. 2008].

\section{ACKNOWLEDGMENTS}

This study was supported by Cochlear Americas. The first author appreciates Bob Shannon and Monita Chatterjee for their advice at the early stage of the study. The authors express warm appreciation to Wendy Parkinson for assisting data collection. Helpful feedback on the initial draft of the manuscript was provided by two anonymous reviewers and the editors, Fan-Gang Zeng and Ruth Anne Eatock.

\section{REFERENCES}

Bierer JA, Middlebrooks JC. Cortical responses to cochlear implant stimulation: channel interactions. J. Assoc. Res. Otolaryngol. 5:32-48, 2004.

Boex C, Kos Mi, Pelizzone M. Forward masking in different cochlear implant systems. J. Acoust. Soc. Am. 114:2058-2065, 2003.

Buchner A, Nogueira W, Edler B, Battmer RD, Lenarz T. Results from a psychoacoustic model-based strategy for the nucleus-24 and freedom cochlear implants. Otol. Neurotol. 29:189-192, 2008.

Cartee LA, Miller CA, van den Honert C. Spiral ganglion cell site of excitation I: comparison of scala tympani and intrameatal electrode responses. Hear. Res. 215:10-21, 2006.

Chatterjee M, Shannon RV. Forward masked excitation patterns in multielectrode electrical stimulation. J. Acoust. Soc. Am. 103: 2565-2572, 1998. 
Eddington DK, Dobelle WH, Brackmann DE, Mladejovsky MG, PARKIN JL. Auditory prostheses research with multiple channel intracochlear stimulation in man. Ann. Otol. Rhinol. Laryngol. 87:1-39, 1978.

EGAN JP, HAKE HW. On the masking pattern of a simple auditory stimulus. J. Acoust. Soc. Am. 22:622-630, 1950.

Hawkins JE, Stevens SS. The masking of pure tones and of speech by white noise. J. Acoust. Soc. Am. 22:6-13, 1950.

Henry BA, Turner CW. The resolution of complex spectral patterns by cochlear implant and normal-hearing listeners. J. Acoust. Soc. Am. 113:2861-2873, 2003.

JesteAdt W, Bacon SP, Lehman JR. Forward masking as a function of frequency, masker level, and signal delay. J. Acoust. Soc. Am. 71:950-962, 1982.

KwOn BJ, van DEN Honert C. Effect of electrode configuration on psychophysical forward masking in cochlear implant listeners. J. Acoust. Soc. Am. 119:2994-3002, 2006.

LevitT H. Transformed up-down methods in psychoacoustics. J. Acoust. Soc. Am. 49:467-477, 1971.

McKay CM, Henshall KR, Farrell RJ, McDermott HJ. A practical method of predicting the loudness of complex electrical stimuli. J. Acoust. Soc. Am. 113:2054-2063, 2003.

MidDLEBRooks JC. Effects of cochlear-implant pulse rate and interchannel timing on channel interactions and thresholds. J. Acoust. Soc. Am. 116:452-468, 2004

Moore BC. Parallels between frequency selectivity measured psychophysically and in cochlear mechanics. Scand. Audiol. Suppl. 25:139-152, 1986.

Moore BC, Glasberg BR. Suggested formulae for calculating auditory-filter bandwidths and excitation patterns. J. Acoust. Soc. Am. 73:1249-1259, 1986a.

Moore BC, Glasberg BR. Comparisons of frequency selectivity in simultaneous and forward masking for subjects with unilateral cochlear impairments. J. Acoust. Soc. Am. 80:93-107, 1986b.
Nelson DA, Donaldson GS. Psychophysical recovery from singlepulse forward masking in electric hearing. J. Acoust. Soc. Am. 109:2921-2933, 2001.

Nelson DA, Donaldson GS, Kreft H. Forward-masked spatial tuning curves in cochlear implant users. J. Acoust. Soc. Am. 123:15221543,2008

Painter T, Spanias A. A review of algorithms for perceptual coding of digital audio signals. Proc. 13th Int. Conf. Digit. Signal Process. 1:179-208, 1997.

SHANNON RV. Two-tone unmasking and suppression in a forwardmasking situation. J. Acoust. Soc. Am. 59:1460-1470, 1976.

SHANnon RV. Multichannel electrical stimulation of the auditory nerve in man. II. Channel interaction. Hear. Res. 12:1-16, 1983.

Smith RL, Chatterjee M, Sanpetrino N. The time course of "simultaneous" masking in cochlear implant listeners: an "overshoot" in electrical stimulation? Abstracts of the 26th annual midwinter research meeting of the Association for Research in Otolaryngology, p. 806, 2003

Stickney GS, Loizou PC, Mishra LN, Assmann PF, Shannon RV, Opie JM. Effects of electrode design and configuration on channel interactions. Hear. Res. 211:33-45, 2006.

TONG YC, CLARK GM. Loudness summation, masking, and temporal interaction for sensations produced by electric stimulation of two sites in the human cochlea. J. Acoust. Soc. Am. 79:19581966, 1986.

White MW, Merzenich MM, Gardi JN. Multichannel cochlear implants: channel interactions and processor design. Arch. Otolaryngol. 110:493-501, 1984.

Zeng FG. Compression and cochlear implants. In: Bacon SP, Fay RR, and Popper AN (ed) Compression from cochlea to cochlear implants, 184-220, 2004.

Zeng FG, Chen H, Han S. Temporal masking in electric hearing. J. Assoc. Res. Otolaryngol. 6:390-400, 2005. 